will decrease without substantial changes to product design, regulation, and/or legislation.

Significance and Contribution to the Field Understanding the patterns of golf cart-related injuries, especially to children and seniors, is critical as their use and injuries are increasing.

\section{MALADAPTIVE MECHANISMS FOR COPING WITH THE DIRECT COSTS OF INJURIES: FOREGOING MEDICAL CARE IN VIETNAM AND KENYA}

Niloufer Taber, Krishna Rao, Cuong Viet Pham, Mohd Fadhli Mohd Yusoff, Abdulgafoor M Bachani. Johns Hopkins International Injury Research Unit, Johns Hopkins University Bloomberg School of Public Health

\subsection{6/injuryprev-2020-savir.126}

Statement of Purpose Uninsured patients may attempt to cope with the costs of an injury through various financial and nonfinancial coping mechanisms. Certain coping mechanisms, such as reducing health care utilization, may minimize direct medical costs while increasing indirect costs.

Methods/Approach The Health, Economic and Long-term Social Impact of Injuries (HEALS) project was a hospital-based longitudinal study of patients conducted in four countries, including Vietnam and Kenya, in 2015-2016. Using baseline survey data, we compared differences in length of hospital stay by health insurance status using a multivariable linear regression, and conducted a multivariable logistic regression to evaluate the relationship between insurance and foregoing additional health care.

Results The sample included 1022 patients in Vietnam and 994 in Kenya. Of these, $56.2 \%$ and $68.8 \%$ lacked insurance and $21.5 \%$ and $3.7 \%$ left against medical advice in Vietnam and Kenya, respectively. In Vietnam, uninsured patients left 2.4 days earlier than those with insurance, controlling for injury severity and mechanism; patient demographics; preinjury household poverty status and the patient's contribution to household income $(\beta=-2.441, \mathrm{p}<0.001)$. Compared to insured patients, uninsured patients in Vietnam had 2.3 times the odds of leaving against medical advice, controlling for the same set of variables. $(\mathrm{OR}=2.327, \mathrm{p}<0.001)$. In Kenya, those without insurance left on average 0.5 days earlier and had over 3 times the odds of leaving against medical advice, compared to insured patients, although neither estimate was statistically significant $(\beta=-0.592, \quad p=0.838$ and $O R=3.190$, $\mathrm{p}=0.124)$.

Conclusions Patients without health insurance may be foregoing health care in order to avoid additional direct health care costs, and in the process increasing indirect costs if leaving against medical advice prolongs a period of disability.

Significance and Contributions to Injury and Violence Prevention Science Improving the breadth of health insurance can protect against both direct and indirect costs of injuries.

\section{EXAMINING THE RELATIONSHIP BETWEEN APP USAGE AND INJURY PREVENTION BEHAVIOR CHANGE}

${ }^{1}$ Elise Omaki, ${ }^{1}$ Wendy Shields, ${ }^{1}$ Eileen McDonald, ${ }^{2}$ Mary Aitken, ${ }^{1}$ Andrea Gielen. ${ }^{1} J o h n s$ Hopkins Center for Injury Research and Policy; ${ }^{2}$ Arkansas Children's Hospital

10.1136/injuryprev-2020-savir.127
Purpose Safety in Seconds (SIS) is a smartphone app that has demonstrated effectiveness at changing injury prevention behaviors. The aim of this analysis was to understand how parents engaged with each component of the app and determine if there was an association between app usage and behaviors.

Methods Parents of children aged 4-7 visiting the pediatric emergency room in the two participating sites (regardless of the reason for the visit) were recruited. Study participants downloaded the SIS app onto their smartphone and were randomized receive car seat or fire safety information. Both groups completed a 10-minute assessment about their safety knowledge and behaviors and received tailored feedback relevant to their study group. The app contained a portal with educational links and sent monthly push notification reminders for parents to interact with the app. Parents completed follow-up assessments at 3-months and 6-months.

Results Among the 602 parents completing all follow up points, 37\% visited each page of the report, 19\% emailed the report to themselves or someone else, and $33 \%$ visited the Parent Portal. Among 555 parents reporting at least one unsafe behavior at baseline, $130(23.5 \%)$ changed their safety behaviors and reported no unsafe behaviors at the 6-month follow up. Those changing their behavior had more mean clicks ( 24 vs. $18 ; \mathrm{p}<0.0001)$, were more likely to have visited all pages of the report $(48 \%$ vs. $32 \% ; \mathrm{p}<0.01)$ and emailed the report $(29 \%$ vs. $15 \% ; \mathrm{p}<0.001)$ than those who did not change their behavior. In a multivariable logistic regression, those visiting all pages of the report (OR 1.58; 95\% CI 1.00, 2.50 ) or emailing the report (OR 1.77; 95\% CI 1.07, 2.94) were more likely to have changed their behavior at six months than those not.

Conclusion Smartphone applications hold potential for changing behaviors that are known to improve child passenger safety.

\section{EXAMINING THE ASSOCIATION BETWEEN REJECTION SENSITIVITY AND AGGRESSION IN MIDDLE SCHOOL STUDENTS}

Mary Cunningham. University of Michigan School of Public Health

\subsection{6/injuryprev-2020-savir.128}

Statement of Purpose As violence remains a persistent problem in schools, it is crucial to better understand factors that predict aggressive behavior in youth. Previous research has shown that rejection can elicit aggressive behavior, particularly in individuals who report high levels of rejection sensitivity. Rejection sensitivity refers to the tendency to expect and overreact to rejection.

Methods/Approach The study sample includes 697 students recruited from middle schools in Miami, FL and Los Angeles, CA. Students completed two surveys on school climate during the 2018-2019 school year as part of a school safety program evaluation. We used linear regression to determine the correlates of aggressive behavior at Time 2. Covariates in the model included rejection sensitivity, age, gender, race, and victimization at Time 2, aggressive behavior at Time 1, location, and school.

Results Findings reveal that higher levels of rejection sensitivity were associated with more engagement in aggressive behavior $(\beta=0.18 ; 95 \%$ CI: $0.01,0.35)$. We also found that Time 1 aggressive behavior $(\beta=0.35 ; 95 \%$ CI: $0.16,0.53)$, age 